Published in final edited form as:

Inflamm Bowel Dis. 2013 ; 19(4): 832-839. doi:10.1097/MIB.0b013e3182802b57.

\title{
Rates and Predictors of Oral Medication Adherence in Pediatric IBD Patients**
}

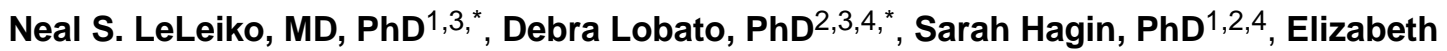 \\ McQuaid, PhD ${ }^{2,3,4}$, Ronald Seifer, PhD $^{2,3,4}$, Sheryl J. Kopel, MSc ${ }^{2,3,4}$, Julie Boergers, \\ PhD $^{2,3,4}$, Jack Nassau, PhD ${ }^{2,3,4}$, Kristina Suorsa, BS ${ }^{1}$, Jason Shapiro, MD ${ }^{1,3}$, and Barbara \\ Bancroft, MS, RN ${ }^{1}$
}

${ }^{1}$ The Division of Pediatric Gastroenterology, Nutrition and Liver Diseases, The Department of Pediatrics, Hasbro Children's Hospital/Rhode Island Hospital, Providence Rhode Island ${ }^{2}$ The Division of Child and Adolescent Psychiatry, Department of Psychiatry, Hasbro Children's Hospital/Rhode Island Hospital, Providence, Rhode Island ${ }^{3}$ The Warren Alpert School of Medicine at Brown University, Providence, Rhode Island ${ }^{4}$ Bradley Hasbro Research Center, Providence, Rhode Island

\begin{abstract}
BACKGROUND \& AIMS-Symptoms of Inflammatory Bowel Disease (IBD) include bloody diarrhea, fatigue, abdominal pain and weight loss. Long-term management of remission for most patients requires adherence to taking one or more oral medications daily, in the absence of symptoms. We investigated whether disease characteristics and behavioral characteristics predict adherence to prescribed medical regimes.
\end{abstract}

METHODS-Patients 8 to 17.5 years of age, newly diagnosed with IBD as well as a matched cohort previously diagnosed were studied over a 6-month period. Adherence was assessed using medication electronic monitoring devices (MEMS); participants and parents completed questionnaires regarding emotional and behavioral functioning, and biological parameters were monitored.

\footnotetext{
** Data from this report was presented in part at a platform session of Digestive Diseases Week (American Gastroenterological Association) May, 2011, Chicago Il, USA.

Corresponding Author: Neal S. LeLeiko MD, PhD, Department of Pediatrics, MPH Rm 134, 593 Eddy Street, Providence, RI 02903, neal_leleiko@brown.edu.

* Dr. LeLeiko and Dr. Lobato contributed equally to this work.

Publisher's Disclaimer: This is a PDF file of an unedited manuscript that has been accepted for publication. As a service to our customers we are providing this early version of the manuscript. The manuscript will undergo copyediting, typesetting, and review of the resulting proof before it is published in its final citable form. Please note that during the production process errors may be discovered which could affect the content, and all legal disclaimers that apply to the journal pertain.

Roles: Study conception, design and implementation: LeLeiko, Lobato, McQuaid, Seifer, Boergers, Nassau

Study acquisition of data, data management, study conduct: Kopel, Suorsa, Bancroft

Study evaluation of data and writing: LeLeiko, Lobato, Kopel, Hagin, McQuaid, Seifer, Boegers, Nassau, Shapiro Statistical analysis: Kopel, Hagin, Lobato, LeLeiko

Manuscript preparation: LeLeiko, Lobato, Hagin, Shapiro, McQuaid

Manuscript approval of final version: LeLeiko, Lobato, Kopel, Hagin, McQuaid, Seifer, Boegers, Nassau, Suorsa, Shapiro, Bancroft

Disclosures: None of the authors have anything to disclose.
} 
RESULTS-Adherence was monitored for 45 newly and 34 previously diagnosed patients. In total, 16,478 patient days (including 12,066 discrete days) were electronically monitored. Overall $70.6 \%$ of 5-ASA and $65.4 \%$ of 6-MP doses were taken. Only $25 \%$ and $15 \%$ of older adolescents took at least $80 \%$ of their 5-ASA and 6-MP, respectively, compared to about $83 \%$ and $64 \%$ of 8 11 year-olds. Only age and behavioral issues were statistically linked to rates of adherence.

CONCLUSIONS-Adherence to commonly prescribed oral medications for IBD is challenging for patients. Screening for emotional and behavioral problems, especially among older adolescents, would be important in identifying patients at risk for poor adherence, who might benefit from interventions. Biological solutions, while critical, when applied without attention to behavioral issues, are not likely to provide the level of therapeutic benefit that can be provided in a combined bio-behavioral approach.

\section{Keywords}

BioBehavioral Model; Medication Event Monitoring Systems; electronic drug monitoring

\section{Introduction}

Inflammatory Bowel Diseases (IBD), mainly Crohn's disease (CD) and ulcerative colitis (UC), are chronic disorders of the gastrointestinal tract. IBD is characterized by remissions and unpredictable relapses of disease often accompanied by bloody diarrhea, fatigue, severe abdominal pain, and chronic weight loss, as well as extra-intestinal manifestations such as arthritis. After inducing clinical remission in IBD, emphasis of management shifts to maintaining symptom-free remission and decreasing the frequency of flares through close adherence to one or more maintenance medications typically taken daily in the absence of symptoms.

The study of adherence to medication has only recently been the subject of concern in pediatric IBD, even though the demands of adherence to daily IBD regimens are perceived as a major burden by children and adolescents ${ }^{1}$. Medication adherence itself may be defined and estimated in different ways. The majority of the studies of adherence define an $80 \%$ cutoff for distinguishing "adherent" from "non-adherent" patients ${ }^{2}$. There is however, no evidence that there is any biological significance to this arbitrary cut-off. The majority of studies of adherence in IBD rely on subjective self-report which yields estimates of "adherence" at around $80 \%{ }^{3,4}$. When more objective methods of adherence are used, such as pill counts, prescription refill counts, and metabolite levels such as 6-thioguanine (6-TGN) and 6-methylmercaptopurine nucleotide (6-MMPN) for thioguanine medications, adherence rates drop to about $22-50 \% \%^{5,6}$. A study of 42 subjects $^{7}$ compared adherence by parent/ patient interview, pill count, and 6-TGN levels. Results from interviews suggested adherence rates of $90 \%$ for 6-MP/azathioprine and $98 \%$ for 5-ASA. The more objective measures, pill count and blood levels, suggested rates of $46 \%$ for $6-\mathrm{MP}$ and $12 \%$ for $5 \mathrm{ASA}^{7}$. Clearly, the methods used to assess adherence have an impact on the estimates of adherence. Electronic medication monitoring systems, the current standard for measuring adherence in other chronic conditions ${ }^{8}$, have not generally been used to study adherence in pediatric IBD. 
In addition to utilizing the most objective methods of measuring adherence over time, it is important to examine the correlates and predictors of adherence in IBD. Doing so would help identify patients at risk for poor adherence and to target adherence interventions accordingly. We know from the study of other pediatric chronic illnesses that poor adherence is related to characteristics of the illness as well as of the child and family ${ }^{9,10}$. Diseases such as IBD that are characterized by an intermittent and variable course and are treated with complex regimens of multiple medications make consistent adherence particularly challenging ${ }^{11}$. Additionally, adherence appears to be worse among patients who have emotional and behavioral problems 9 . This is a particularly relevant consideration in IBD since there is a well-established association between IBD and psychological distress among children ${ }^{12}$ and adults. ${ }^{13,14}$

The primary aim of the current study was to examine adherence to maintenance medications 5-ASA and 6-MP among children and adolescents with IBD, utilizing electronic monitoring technology over a 6-month period. The second aim was to identify biomedical (e.g., diagnosis, IBD symptoms, disease activity, time since diagnosis), treatment regimen (5ASA, 6-MP or both), psychological (e.g., behavioral, anxiety, and depressive symptoms), and individual (age, gender) characteristics associated with adherence. We hypothesized that one or some combination of these biomedical, psychological and individual characteristics, present at the time of enrollment into our study, might identify patients at higher risk for poor medication adherence.

\section{Methods}

\section{Recruitment}

Patients 8-17.5 years of age were recruited for the current study at Hasbro Children's Hospital in Providence, Rhode Island, if they had a diagnosis of Crohn's Disease (CD) or Ulcerative Colitis (UC) confirmed by endoscopic, pathologic, or radiographic findings. Two groups of patients were recruited - newly diagnosed (enrolled within 30 days of their diagnosis) and previously-diagnosed patients who were at least one year past their initial diagnosis, and matched to newly-diagnosed patients on gender, diagnosis (CD v. UC), and age. Inclusion of previously-diagnosed patients allowed us to examine the potential contribution of disease chronicity on adherence. All patients were co-enrolled in the Pediatric IBD Collaborative Research Group Registry ("Registry"), an ongoing prospective, observational, biomedical research registry being conducted at 24 pediatric gastroenterology centers in the United States and Canada collectively known as the Pediatric Inflammatory Bowel Disease Collaborative Research Group. Standardized medical data for each patient enrolled in the Registry is prospectively collected and submitted to a centralized data management center at the time of diagnosis, 30 days post diagnosis, and quarterly thereafter. Patients are managed according to the dictates of their physicians, not by standardized protocols. Biomedical clinical data for our subjects, from the Registry, were analyzed as part of the current study. All patients and their parents provided informed assent and consent prior to participating in the study, which was approved by the hospital Institutional Review Board. 


\section{Procedures and Measures}

Biomedical and behavioral measures were collected at the time of study enrollment.

Electronic monitoring of adherence to IBD medications 5-ASA and 6-MP was conducted for up to 6 months following enrollment.

Electronic Monitoring of Adherence-Patient daily adherence to oral medications (5ASA and 6-MP) was monitored electronically using the Medication Event Monitoring Systems ("MEMS 6 TrackCap") (Aardex Group, Sion, Switzerland) [MEMS]. This device consists of a medication-containing bottle fitted with a special "TrackCap". The "TrackCap" contains an integral electronic micro-circuit that records the time and date of each opening and closing of the container. Families were informed that the electronic monitoring device recorded the time and date of each opening of the bottle. In other areas of investigation, MEMS are strongly correlated with serum assays, pharmacy-refill data ${ }^{15,16}$, and clinical laboratory outcomes ${ }^{17}$.

For participants taking both 5-ASA and 6-MP, two MEMS were assigned and adherence rates were calculated separately for each medication. "TrackCap" Adherence (TCA) was defined as the number of times the bottle was opened divided by the number of expected openings, based on prescriptions documented in the medical records. Patterns of medication intake were inquired about during monthly contact calls and expected doses were adjusted to account for routines and events that may impact adherence rates such as the use of weekly pill organizers or vacations and inpatient hospitalizations when MEMS devices were unavailable.

Socio-demographics-Parents reported household composition and income, race and ethnicity, and parents' marital status, education, and occupation (Table 1).

Biomedical Factors-Data regarding patient diagnosis (CD v. UC), date of diagnosis, disease activity, and IBD symptoms (pain, bleeding, and bowel functioning) at time of enrollment were obtained from the PIBDRC registry data base. Disease status of previously diagnosed patients at the time of their diagnosis was also obtained from the PIBDRC registry database.

\section{IBD Disease Activity}

The Abbreviated Pediatric Crohn's Disease Activity Index (abbPCDAI ${ }^{20}$ : assessed disease activity in children with CD. The abbPCDAI sums 6 items from the PCDAI ${ }^{18,19,21}$ assessing pain, general well-being, number of stools per day, abdominal tenderness, perirectal disease, and weight. Laboratory measures are not included. The abbPCDAI score ranges from 0-60: an activity score of $<15=$ inactive disease; $15-25=$ mild; $>25=$ moderatesevere. The abbPCDAI has been found to be a reliable and valid measure of disease activity with correlations between abbPCDAI and PCDAI ranging from .68 to .85 and between the abbPCDAI and Physician's Global Assessment (PGA) reported as .64. ${ }^{18,19,20,22}$

The Pediatric Ulcerative Colitis Activity Index (PUCAI) ${ }^{23}$ : assessed disease activity in children with UC via 6 history items (abdominal pain, rectal bleeding, stool consistency, 
number of stools per 24 hours, nocturnal bowel movements causing wakening, activity restriction). Disease activity scores range 0 to 85 : $<10=$ inactive; $10-34=$ mild, $35-$ $64=$ moderate, and $\geq 65=$ severe.

IBD symptoms-Specific IBD-related symptoms common to CD and UC (e.g., abdominal pain, bowel functioning) were pulled from the abbPCDAI and PUCAI to evaluate the relationship between specific symptoms and adherence across IBD diagnoses. Scores on specific items from the abbPCDAI and PUCAI which reflect patient reported abdominal pain and stools per day were combined to create the variables "pain" and number of stools per day". Due to item categorization inconsistencies across measures (i.e.PUCAI item has 4 categories for stools per day and abb PCDAI item only has 3 categories), scores on these items were first dichotomized and then combined. Scores in the lowest category of each item were considered asymptomatic and scores greater than the lowest category were considered symptomatic. The variable of the PUCAI which reflects patient report of rectal bleeding was examined separately to assess the relationship between adherence and rectal bleeding.

Psychological Functioning-Participants reported on patient emotional and behavioral functioning with the following well-established measures:

Child Behavior Checklist for Ages 6 to 18 (CBCL) ${ }^{24}$ : Parents completed the $C B C L$ to assess children's global emotional and behavior problems. The CBCL consists of 113 items describing different behavior problems that are rated on a scale from 0 (not true) to 2 (very or often true). Higher scores reflect greater emotional and behavioral problems. Three composite T-scores are derived assessing Internalizing (e.g. anxiety, depressed mood), Externalizing (e.g. argumentative, aggressive), and Total Behavior Problems.

Children's Depression Inventory (CDI) ${ }^{25}$ : The $C D I$ is a 27 item self-report measure of depressive symptoms in children ages 7-17 years. Children rate items on a range from 0 (Absence of symptom) to 2 (Definite symptom). Higher Total scores indicate greater depressive symptom severity.

Multidimensional Anxiety Scale for Children (MASC) ${ }^{\mathbf{2 6}}$ : The $M A S C$ is a 39-item selfreport measure that assesses anxiety symptoms. Children rate items on a 4-point Likert scale from 0 ("never true about me") to 3 ("often true about me"). The MASC yields a Total Anxiety T- Score, with higher scores indicating greater symptom severity.

\section{Statistical Analyses}

All analyses were conducted using statistical package SPSS, (Windows version 17; SPSS Inc, Chicago, Il). Preliminary analyses comparing cohort groups utilized ANOVA and chisquare analyses as appropriate. ANOVAs and Scheffe post-hoc tests were used to evaluate differences in TCA rates within the sample. Correlation coefficients were computed to evaluate relationships between biomedical and behavioral health variables and TCA. Regression analyses were conducted to evaluate the impact of biomedical and behavioral health variables on TCA rate. 


\section{Results}

Participants

Participants were 45 newly-diagnosed patients and 34 previously-diagnosed patients. As seen in Tables 1 and 2, there were no differences between the newly- and previouslydiagnosed groups in demographic characteristics at the time of enrollment or disease activity at the time of diagnosis. Overall, the patients were from two-parent families with high levels of education, employment, and income. As expected, at the time of enrollment into our study the newly-diagnosed patients had more active disease than previously-diagnosed patients who were on average 42 months $(\mathrm{SD}=16.9)$ from the time of their diagnosis and more likely to be in remission.

\section{Length of monitoring period and dosing schedule}

The mean duration of electronic monitoring for the 64 participants prescribed 5-ASA was $170.5(\mathrm{SD}=57)$ days and for the 36 participants prescribed 6-MP was $154.7(\mathrm{SD}=66.8)$ days. Twenty-seven participants were monitored for both drugs at some point during the study. Of those 27 participants, twenty-one were monitored for both 5-ASA and 6-MP simultaneously for an average of $162.8(\mathrm{SD}=71.1)$ days (Table 5). The majority of patients were prescribed to take 5-ASA twice per day (92\%) and 6-MP once per day (94.4\%).

\section{Adherence Estimates}

TCA rates first were examined both by the overall proportion of prescribed medication doses taken. As shown in Table 3, TCA among participants averaged almost 71\% for 5-ASA and nearly $65 \%$ for $6-\mathrm{MP}$ over the 6 month tracking period. Overall TCA rates varied widely across participants, ranging from 0 to 100 percent.

\section{Adherence by Patient Groups}

As shown in Table 3, preliminary analyses revealed no differences in medication adherence rates by sex or diagnosis (CD v UC). Age was categorized into 3 groups: pre-adolescent $(8<12$ years old), young adolescent $(12<15$ years old), and older adolescent $(15<18$ years old). TCA rates differed significantly by age category for 5-ASA. Post-hoc analyses revealed participants 15 years or older had significantly lower 5-ASA TCA rates compared to younger participants, both those in the $8<12$ year old group (Mean Difference 31.42; $\mathrm{p}=$. 001 ) and those in the $12<15$ year old group (Mean Difference $22.15 ; \mathrm{p}=.03$ ). There was no difference in 5-ASA TCA rates between the $8<12$ year olds and the $12<15$ year olds (Mean Difference 9.28; $\mathrm{p}=.55$ ). TCA rates did not differ by age category for 6-MP; however, the pattern of 6MP adherence was similar to 5ASA adherence with participants 15 years and older having lower average 6MP TCA rates compared to younger participants.

TCA rates differed significantly by cohort (newly v. previously diagnosed) for 5-ASA ( $\mathrm{F}(1$, $62)=4.33, \mathrm{p}=.04)$ but not for 6-MP $(\mathrm{F}(1,34)=0.33, \mathrm{p}=.57)$. Follow-up ANCOVA, controlling for age, was conducted and the difference between cohort groups was no longer significant for 5-ASA $(F(1,61)=1.41, \mathrm{p}=.24)$ cohort (newly vs previously diagnosed), and number of medications (5-ASA only, 6-MP only, and combined). 
TCA rates for 5-ASA, but not $6 \mathrm{MP}$, differed by number of medications monitored. Followup ANCOVA, controlling for age, was conducted and the difference between those who were monitored for 1 versus 2 medications remained $(\mathrm{F}(1,61)=6.31, \mathrm{p}=.015)$ with number of monitored medications accounting for $9 \%$ and age accounting for $21 \%$ of the variance in 5ASA TCA. Average 5-ASA TCA was 77\% in participants monitored for 5-ASA alone compared to $58 \%$ in those monitored for both 5-ASA and 6-MP.

After examining overall proportions of doses taken we then examined whether the patients' overall proportion fell above or below the accepted medication adherence cut-off ( $80 \%$ adherence rate). As shown in Figure 1, over the 6 month period of our study, $45 \%$ of participants accessed their 5-ASA medications less than $80 \%$ of the prescribed times, and $58 \%$ of participants accessed their 6-MP medications less than $80 \%$ of prescribed times. Figure also depicts different rates of adherence by age. Seventy-five percent of older adolescents opened their 5-ASA bottles less than $80 \%$ of the number of times prescribed and $85 \%$ of them opened their 6-MP bottles less than $80 \%$ of the number of times prescribed.

Given the similar adherence rates between cohorts, sex, and diagnosis, groups were combined in subsequent analyses, controlling for age and number of medications as necessary.

Biomedical and Behavioral Factors and Track Cap Adherence-The relationship between biomedical and behavioral factors at the time of enrollment and overall TCA for 5ASA and 6-MP were examined separately. Given the relationship between age and medication adherence, partial correlations were computed among the biomedical and behavioral variables and TCA scores holding age constant. Partial correlations between TCA, PGA, abbPCDAI, PUCAI, and IBD-related symptoms were not significant (Table 4). CBCL Total and Externalizing and Internalizing sub-domains, MASC, and CDI scores were correlated with TCA scores for both 5-ASA and 6-MP. Results indicated that higher CBCL scores and subscales were associated with poorer overall 5-ASA and 6-MP TCA while controlling for age. Partial correlations between MASC and CDI scores and TCA were not significant (Table 4).

Sequential regression analyses were conducted to examine the relative contributions of biomedical and psychological factors on TCA. PGA and diagnosis (CD v. UC) were entered in the first step and number of monitored medications was entered in the second step. Age was entered in the third step. The associated behavioral health variable, CBCL Total, was entered in the final step.

The first set of predictors, PGA and diagnosis, did not account for a significant proportion of variance in 5-ASA TCA, $\mathrm{R}^{2}=.006, \mathrm{~F}(2,47)=.15, \mathrm{p}=.86$. The addition of number of monitored medications did not account for a significant proportion of 5-ASA TCA, $\mathrm{R}^{2}$ change $=.04, \mathrm{~F}_{\text {inc }}(1,46)=1.83, \mathrm{p}=.18$. The addition of age to the equation resulted in a significant increment in $\mathrm{R}^{2}$, with age accounting for $20 \%$ of the variance in 5-ASA TCA, $\mathrm{F}_{\text {inc }}(1,45)=11.66, \mathrm{p}=.001$. Finally, the addition of CBCL total accounted for a significant proportion (13\%) of the variance in 5-ASA TCA after controlling for biomedical factors and age, $F_{\text {inc }}(1,44)=9.32, \mathrm{p}=.004$. 
Results for 6-MP TCA showed a trend similar to the 5-ASA TCA patterns but none of the biomedical or behavioral factors accounted for a significant proportion of the variance in 6MP TCA. Results for step 1 (PGA and diagnosis), $\mathrm{R}^{2}=.008, \mathrm{~F}(2,24)=0.95, \mathrm{p}=.91$ and step 2 (number of monitored medications), $\mathrm{R}^{2}$ change $=.014, \mathrm{~F}_{\text {inc }}(1,23)=0.33, \mathrm{p}=.57$, were not significant. The addition of age in the third step was not significant, $\mathrm{R}^{2}$ change $=.08, \mathrm{~F}_{\text {inc }}(1$, $22)=1.96, p=.18$. The addition of CBCL total was not significant, $\mathrm{R}^{2}$ change $=.14$, $\mathrm{F}_{\text {inc }}(1,21)=3.92, \mathrm{p}=.06$.

\section{Discussion}

This study examined adherence to maintenance medications 5-ASA and 6-MP among children and adolescents with IBD over approximately a 6-month period (Table 5) using objective electronic methods. Our results indicate that adherence to existing treatments is very difficult for many pediatric patients. Using MEMS technology, we found a wide range in adherence rates, with some patients apparently never taking their medications and others taking it as prescribed $90-100 \%$ of the time. Only patient age and emotional and behavioral functioning made substantial contributions toward predicting adherence to 5-ASA, findings that are consistent with studies of adherence in other pediatric illnesses ${ }^{10,27}$. This pattern was also suggested for adherence to 6-MP regimens, although tests did not reach statistical significance.

Given the debilitating nature of IBD symptoms, we expected that disease activity and associated symptoms at the time of enrollment into our study would be associated with increased TCA; however, neither disease severity nor the type and frequency of biological symptoms at enrollment were associated with overall medication adherence during the subsequent 6 months. It is possible that the effects of disease activity or symptoms on medication taking behavior may have been attenuated over the prolonged monitoring period, especially among the previously diagnosed patients who generally had inactive or mild disease at the time of study enrollment.

Older adolescents (patients often seen by adult gastroenterologists), appear to be at greatest risk for poor adherence, taking at least $80 \%$ of their 5-ASA as prescribed only $25 \%$ of the time and $15 \%$ of the time for 6-MP. Patients, under 12 years of age, had better medication adherence relative to teens, which may be due to greater reliance on parents to administer the medications. However, there still remains a substantial number of young children with poor adherence. Clearly, interventions to improve adherence will not only need to focus on patients but also their caregivers.

\section{Limitations}

There are limitations to the present study. Our medication adherence rates derived from MEMS monitoring may represent an overestimate of adherence for a few reasons. First, one may expect that patients who enroll in a study involving an assessment of medication use who are aware of being monitored may demonstrate somewhat inflated adherence rates. Adherence estimates from the present study suggest that monitoring alone does not eliminate the problem of poor adherence. Second, MEMS devices can only document the times that the medication bottle was opened; they cannot document actual ingestion or if the 
appropriate number of pills was taken. We suspect that the greater the number of prescribed pills per dose, the more likely we were to err on the side of overestimating adherence. This would primarily affect our 5-ASA data because 5-ASA is typically prescribed as multiple, large pills taken multiple times per day (as opposed to 6-MP, which was generally prescribed as fewer and smaller pills taken once per day). Finally, if parents reported that the MEMS bottle with the electronic cap was not used for any understandable reason (e.g., did not want to take the expensive device on a family vacation), we excluded those days from our calculations of adherence. This may have also skewed our results toward higher rates of adherence. Finally, the demographic characteristics of our sample (being predominantly Caucasian, two-parent families with high education and income) are associated with better adherence as compared to patients from underrepresented minorities and disadvantaged neighborhoods ${ }^{28}$. Further research with these groups is needed.

Our results demonstrate that poor adherence to prescribed medication is common in pediatric IBD patients and relevant to clinical practice. Current clinical practice generally relies on self-report, which tends to over-estimate adherence. The extent of adherence is very likely to affect the efficacy of the treatment. Poor adherence poses the risk of increasing dosage to a toxic level or needlessly (or prematurely) stepping up therapy, when what is actually needed is better adherence to the original treatment.

Given the potential impact of poor adherence on clinical care and health outcomes, developing strategies to identify those at risk for poor adherence as well as developing interventions to increase adherence is essential. Based on our data, screening for emotional and behavioral problems, especially among older adolescents, would be an important first step in targeting intervention resources to the highest risk groups.

Results from the present study have many important implications for future research. Clinical trials in pediatrics rarely include adherence as an inclusion criteria for studies of medication efficacy. Clinically, it is obvious that being unable to identify patients who are not taking medications as prescribed, severely hampers the selection and implementation of optimal therapeutic options. Conversely, if we are able to identify characteristics that predict adherence to therapy or to alter behavior to improve adherence, then the increased number of potentially effective therapies available may improve outcomes. Given the need for longterm medication intake to prevent relapse, identifying an adherence threshold necessary for medication efficacy would be highly useful for clinical practice.

While it is obvious that more work needs to be done in this area, it is also obvious that successful management of pediatric patients, especially those who might not be responding as we might expect, may benefit from the assessment and possible intervention from behavioral health providers. Biological solutions, although critical, when applied without attention to behavioral issues, are unlikely to provide the level of therapeutic benefit to our patients that can be provided in a combined biological/behavioral approach.

\section{Acknowledgments}

Funding: This work was funded by NIH R21 HDO58828 


\section{References}

1. Loonen HJ, Derkx BHF, Griffiths A. Pediatricians overestimate importance of physical symptoms upon children's health concerns. Medical Care. 2002; 40:996-1001. [PubMed: 12395031]

2. Hommel KA, Davis CM, Baldassano RN. Objective versus subjective assessment of oral medication adherence in pediatric inflammatory bowel disease. Inflamm Bowel Dis. 2009; 15:589-93. [PubMed: 18985746]

3. Reed-Knight B, Lewis JD, Blount RL. Association of disease, adolescent, and family factors with medication adherence in pediatric inflammatory bowel disease. J Pediatr Psychol. 2011; 36:308-17. [PubMed: 20798185]

4. Bernal I, Domenech E, Garcia-Planella, et al. Medication-taking behavior in a cohort of patients with inflammatory bowel disease. Digestive Disease Science. 2006; 12:2165-2169.

5. Trindade, Arvind J., Ehrlich, A., et al. Are your patients taking their medicines? Validation of a new adherence scale in patients with inflammatory bowel disease and comparison with physical perception of adherence. Inflamm Bowel Dis. 2011; 17:599-604. [PubMed: 20848512]

6. Hommel KA, Davis CM, Baldassano R. Medication adherence and quality of life in pediatric inflammatory bowel disease. J Pediatr Psychol. 2008; 33:867-874. [PubMed: 18337262]

7. Hommel KA, Davis CM, Baldassano RN. Objective versus subjective assessment of oral medication adherence in pediatric inflammatory bowel disease. Inflamm Bowel Dis. 2009; 15:589-593. [PubMed: 18985746]

8. Ingerski LM, Hente EH, Avani C, et al. Electronic Measurement of Medication Adherence in Pediatric Chronic Illness: A Review of Measures. J Peds. 2011; 159:528-534.

9. Drotar D, Bonner MS. Influences on adherence to pediatric asthma treatment: a review of correlates and predictors. Journal of Developmental and Behavioral Pediatrics. 2009; 30:574-582. [PubMed: 19996903]

10. McQuaid EL, Kopel SJ, Klein RB, et al. Medication adherence in pediatric asthma: reasoning, responsibility, and behavior. J Pediatr Psychol. 2003; 28:323-33. [PubMed: 12808009]

11. Matsui, DM. Children's adherence to medication treatment. In: Drotar, D., editor. Promoting adherence to medical treatment in chronic childhood illness: Concepts, methods, and interventions. Mahway, NH: Lawrence Erlbaum Associates, Publishers; 2000. p. 135-152.

12. Mackner LM, Crandall WV. Oral medication adherence in pediatric inflammatory bowel disease. Inflamm Bowel Dis. 2005; 11:1006-1012. [PubMed: 16239847]

13. North CS, Hong BA, Alpers DH. Relationship of functional gastrointestinal disorders and psychiatric disorders: implications for treatment. World J Gastroenterol. 2007; 13:2020-7. [PubMed: 17465442]

14. Levenstein S, Prantera C, Varvo V, et al. Psychological stress and disease activity in ulcerative colitis: a multidimensional cross-sectional study. Am J Gastroenterol. 1994; 89:1219-25. [PubMed: 8053438]

15. Farley J, Hines S, Musk A, et al. Assessment of adherence to antiviral therapy in HIV-infected children using the Medication Event Monitoring System, pharmacy refill, provider assessment, caregiver self report, and appointment keeping. J Acquir Immune Defic Syndr. 2003; 33:211-8. [PubMed: 12794557]

16. Gerson AC, Furth SL, Neu AM, Fivush BA. Assessing associations between medication adherence and potentially modifiable psychosocial variables in pediatric kidney transplant recipients and their families. Pediatr Transplant. 2004; 8:543-50. [PubMed: 15598321]

17. Martin S, Elliott-DeSorbo DK, Wolters PL, et al. Patient, caregiver and regimen characteristics associated with adherence to highly active antiretroviral therapy among HIV-infected children and adolescents. Pediatr Infect Dis J. 2007; 26:61-7. [PubMed: 17195708]

18. Kappelman MD, Crandall WV, Colletti RB, et al. Short pediatric Crohn's disease activity index for quality improvement and observational research. Inflamm Bowel Dis. 2011; 17(1):112-7.

[PubMed: 20812330] 
19. Turner D, Griffiths AM, Walters TD, et al. Mathematical weighting of the pediatric Crohn's disease activity index (PCDAI) and comparison with its other short versions. Inflamm Bowel Dis. 2012; 18(1):55-62. [PubMed: 21351206]

20. Shepanski MA, Markowitz JE, Mamula P, et al. Is an abbreviated Pediatric Crohn's Disease Activity Index better than the original? J Pediatr Gastroenterol Nutr. 2004; 39:68-72. [PubMed: 15187784]

21. Hyams J, Ferry G, Mandel F, et al. Development and validation of a Pediatric Crohn's Disease Activity Index. J Pediatr Gastroenterol Nutr. 1991; 12:439-447. [PubMed: 1678008]

22. Loonen HJ, Griffiths AM, Merkus MP, et al. A critical assessment of items on the Pediatric Crohn's Disease Activity Index. J Pediatr Gastroenterol Nutr. 2003; 36(1):90-5. [PubMed: 12500002]

23. Turner D, Hyams J, Markowitz J, et al. Appraisal of the pediatric ulcerative colitis activity index (PUCAI). Inflamm Bowel Dis. 2009 Aug; 15(8):1218-23. [PubMed: 19161178]

24. Achenbach, TM., Rescorla, LA. Manual for the ASEBA School-Age Forms and Profiles. Burlington, VT: University of Vermont, Research Center for Children, Youth, and Families; 2001.

25. Kovacs, M. The Children's Depression Inventory. New York: Multi-Health Systems; 1992.

26. March JS, Parker JD, Sullivan K, et al. The Multidimensional Anxiety Scale for Children (MASC): factor structure, reliability, and validity. Journal of the American Academy of Child \& Adolescent Psychiatry. 1997; 36:554-565. [PubMed: 9100431]

27. McQuaid EL, Everhart RS, Seifer R, Kopel SJ, Koinis Mitchell D, Klein RB, Esteban CA, Fritz GK, \& Canino G. Medication adherence among Latino and Non-Latino White children with asthma. Pediatrics. (in press).

28. Rohan J, Drotar D, McNally K, et al. Adherence to pediatric asthma treatment in economically disadvantaged African-American children and adolescents: an application of growth curve analysis. J Pediatr Psychol. 2010; 35:394-404. [PubMed: 19710251] 


\section{Panels $A$ and $B$}

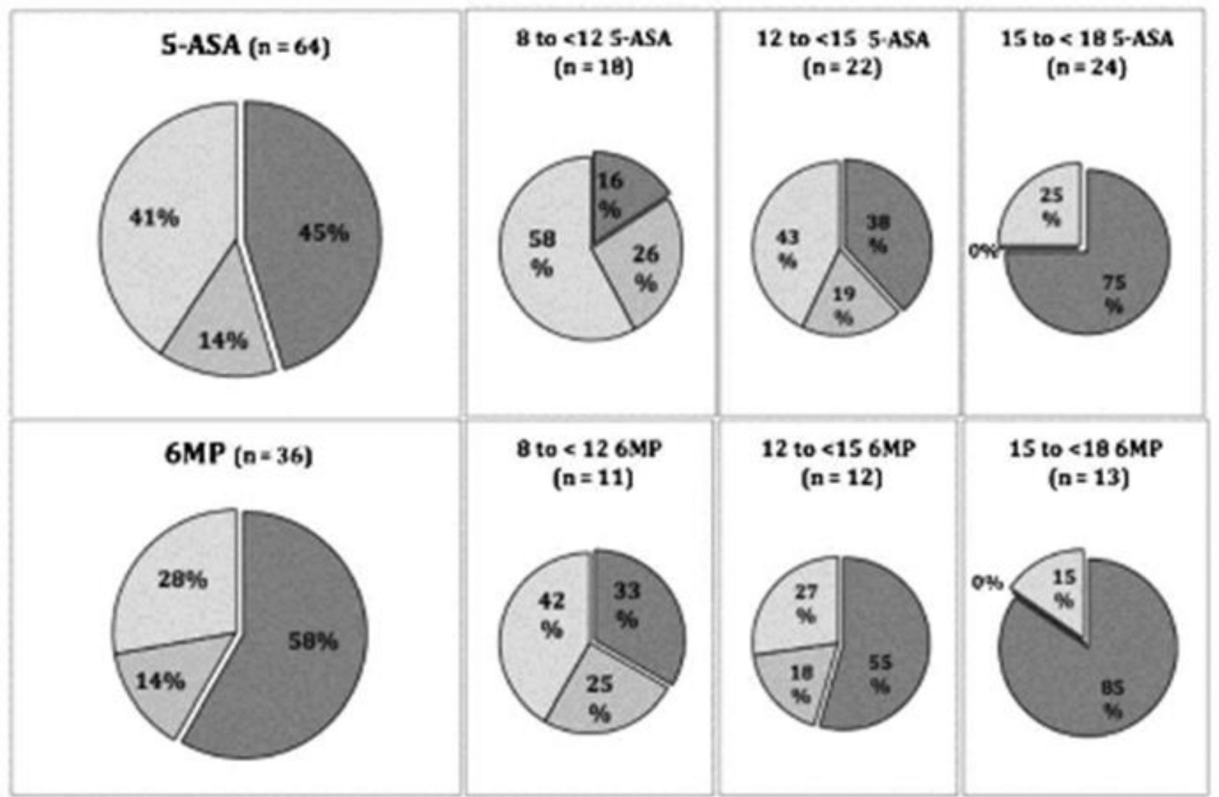

$91 \%-100 \%$ Adherence

B1\% - 90\% Adherence

$<80 \%$ Adherence

\section{Panel C}

\begin{tabular}{|c|c|c|c|c|c|}
\hline \%TrackCap Adherence & $\begin{array}{l}91-100 \% \\
\text { Adherence }\end{array}$ & $\begin{array}{c}81-90 \% \\
\text { Adherence }\end{array}$ & $\begin{array}{r}-50 \%-80 \% \\
\text { Adherence }\end{array}$ & $\begin{array}{c}-25 \%-\sim 50 \% \\
\text { Adherence }\end{array}$ & $\begin{array}{c}<-25 \% \\
\text { Adherence }\end{array}$ \\
\hline \multicolumn{6}{|l|}{ 5-ASA } \\
\hline All ages combined & $40.6 \%$ & $14.1 \%$ & $21.9 \%$ & $9.4 \%$ & $14.1 \%$ \\
\hline$\%$ of paticnts $<12 y$ & $57.9 \%$ & $26.3 \%$ & $5.3 \%$ & $10.5 \%$ & $0.0 \%$ \\
\hline$\%$ of patients $12<15 y$ & $42.9 \%$ & $19.0 \%$ & $23.8 \%$ & $4.8 \%$ & $9.5 \%$ \\
\hline$\%$ of patients $15+y$ & $25.0 \%$ & $0.0 \%$ & $33.3 \%$ & $12.5 \%$ & $29.2 \%$ \\
\hline \multicolumn{6}{|l|}{ 6-MP } \\
\hline All ages combined & $27.8 \%$ & $13.9 \%$ & $30.6 \%$ & $13.9 \%$ & $13.9 \%$ \\
\hline $\begin{array}{r}\% \text { of patients under } 12 \\
\text { years old }\end{array}$ & $41.7 \%$ & $25.0 \%$ & $16.7 \%$ & $16.7 \%$ & $0.0 \%$ \\
\hline $\begin{array}{r}\% \text { of patients } 12 \text { to under } \\
15 \text { years old }\end{array}$ & $27.3 \%$ & $18.2 \%$ & $45.5 \%$ & $0.0 \%$ & $9.1 \%$ \\
\hline $\begin{array}{r}\% \text { of patients } 15 \text { years old } \\
\text { and older }\end{array}$ & $15.4 \%$ & $0.0 \%$ & $30.8 \%$ & $23.1 \%$ & $30.8 \%$ \\
\hline
\end{tabular}

Figure 1.

Percentage of Patients at Different Rates of Adherence by Medication and Age:

Panel A: 5-ASA adherence: slices represent percentage of subjects in each grouping (all patients, 8 to 12 year olds, 12 to 15 year olds and older than 15 years) who take greater than $90 \%$ of their prescribed doses, between 80 and $90 \%$ of their prescribed doses, and less than $80 \%$ of their prescribed doses.

Panel B: 6-MP adherence: slices represent percentage of subjects in each grouping (all patients, 8 to 12 year olds, 12 to 15 year olds and older than 15 years) who take greater than 
$90 \%$ of their prescribed doses, between 80 and $90 \%$ of their prescribed doses, and less than $80 \%$ of their prescribed doses.

Panel C: Breakdown of all groups' adherence, by same age groupings, but showing details of adherence in the age groups at different levels of overall adherence.

Note that as groups get older, adherence drops and percentage of patients taking less than $25 \%$ of doses markedly increases. 


\section{TABLE 1}

Patient Sociodemographic Characteristics at Time of Enrollment

\begin{tabular}{|c|c|c|c|c|}
\hline & Newly Diagnosed ( $N=45)$ & Previously Diagnosed $(\mathrm{N}=34)$ & Statistics & $P$ \\
\hline Age, mean (SD), yr & $13.3(2.4)$ & $14.4(3.1)$ & $\mathrm{F}(1,76)=3.28$ & 0.07 \\
\hline Male, $\%$ & 50 & 53 & $\chi^{2}(1, N=78)=0.07$ & 0.80 \\
\hline Diagnosis, $\%$ & & & $\chi^{2}(1, \mathrm{~N}=78)=1.00$ & 0.32 \\
\hline $\mathrm{CD}$ & 82 & 82 & & \\
\hline $\mathrm{UC}$ & 18 & 18 & & \\
\hline Race, $\%$ & & & $\chi^{2}(4, N=78)=5.67$ & 0.26 \\
\hline Caucasian & 91 & 79.4 & & \\
\hline African American & 2.3 & 5.9 & & \\
\hline American Indian & 2.3 & & & \\
\hline Mixed/other & 4.5 & 14.7 & & \\
\hline Latino ethnicity & 10.5 & 12.5 & & \\
\hline \multicolumn{5}{|l|}{ Household } \\
\hline Parents—living together, $\%$ & 74 & 75 & $\chi^{2}(3, \mathrm{~N}=76)=1.27$ & 0.74 \\
\hline Mean maternal education (SD), yr & $14.5(2.1)$ & $14.4(2.1)$ & $F(1,75)=0.03$ & 0.86 \\
\hline Full-time employment, $\%$ & 84 & 94 & $\chi^{2}(3, N=69)=2.33$ & 0.51 \\
\hline Median annual income (SD) & $\$ 78,749(30,023)$ & $\$ 87,499(30,981)$ & $F(1,66)=0.21$ & 0.65 \\
\hline
\end{tabular}


TABLE 2

Patient Biomedical Characteristics at Time of Diagnosis and Time of Enrollment

\begin{tabular}{lccrc}
\hline Disease Activity & Newly Diagnosed & Previously Diagnosed & F & $\boldsymbol{P}$ \\
\hline Time of diagnosis & & & & \\
PGA, mean (SD) & $2.8(0.9)$ & $3.1(0.6)$ & 2.36 & 0.13 \\
abbPCDAI, mean (SD) & $14.2(12.8)$ & $19.4(8.3)$ & 3.21 & 0.08 \\
PUCAI, mean (SD) & $39.1(22.7)$ & $56.7(33.3)$ & 1.19 & 0.30 \\
Time of enrollment & & & & \\
PGA, mean (SD) & $2.6(1.0)$ & $1.3(0.6)$ & 29.48 & 0.00 \\
abbPCDAI, mean (SD) & $11.7(12.1)$ & $3.3(5.0)$ & 10.18 & 0.00 \\
PUCAI, mean (SD) & $35.9(25.7)$ & $8.0(10.4)$ & 5.34 & 0.04 \\
\hline
\end{tabular}

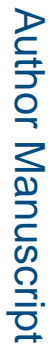

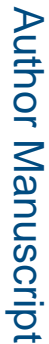




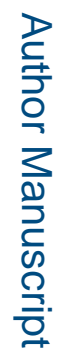
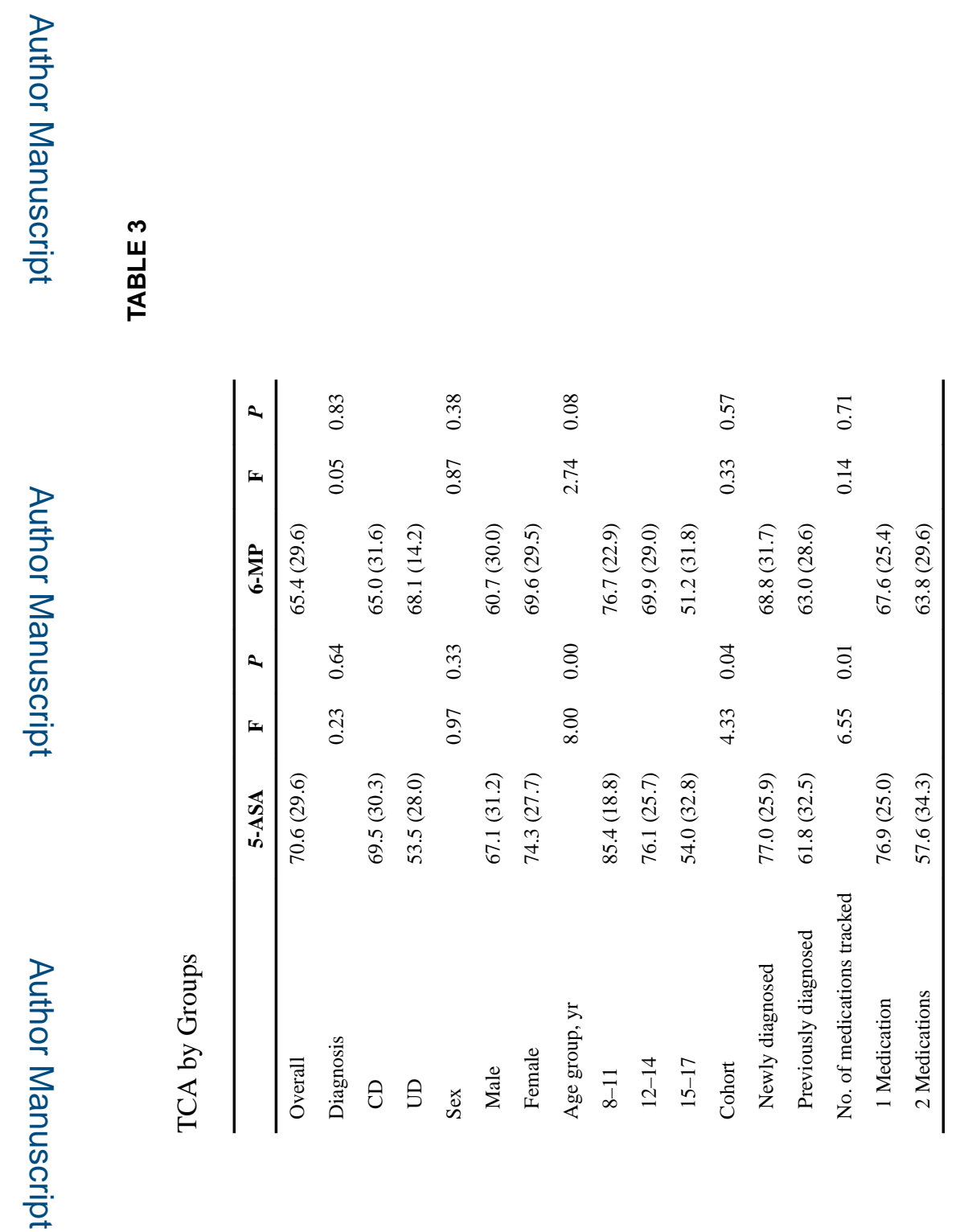

Inflamm Bowel Dis. Author manuscript; available in PMC 2017 November 28. 


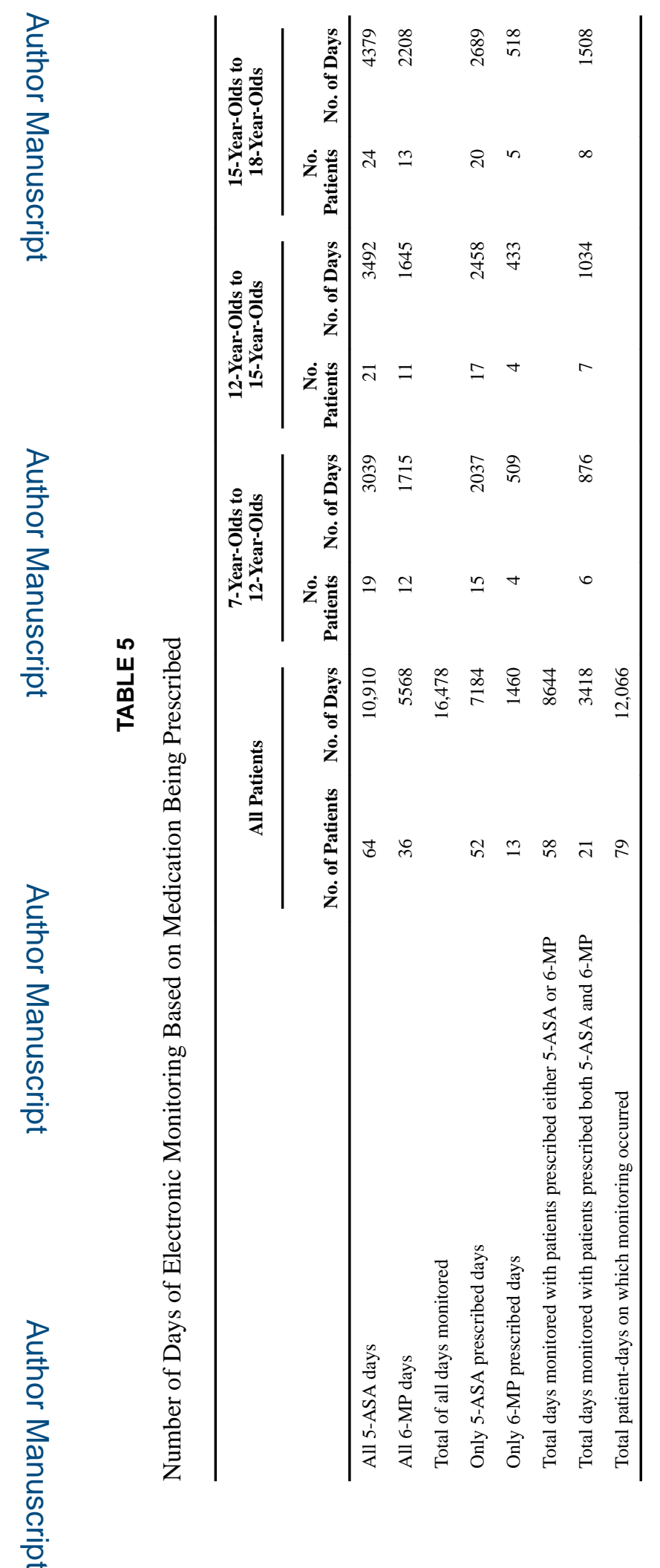

Inflamm Bowel Dis. Author manuscript; available in PMC 2017 November 28. 